\title{
Neuroimaging of aggressive and violent behaviour in children and adolescents
}

\author{
Philipp Sterzer ${ }^{1,2 *}$ and Christina Stadler ${ }^{3}$ \\ Department of Psychiatry, Campus Charité Mitte, Charité - Universitätsmedizin Berlin, Berlin, Germany \\ 2 Berlin School of Mind and Brain, Berlin, Germany \\ ${ }^{3}$ Department of Child and Adolescent Psychiatry, Johann Wolfgang Goethe University, Frankfurt am Main, Germany
}

\section{Edited by:}

Andreas Meyer-Lindenberg, Central Institute of Mental Health, Germany

\section{Reviewed by:}

Essi Viding,

University College London, UK

Joshua W. Buckholtz,

Vanderbilt University, USA

${ }^{*}$ Correspondence:

Philipp Sterzer, Department of Psychiatry, Campus Charité Mitte,

Charité - Universitätsmedizin Berlin,

Charitéplatz 1, D-10117 Berlin,

Germany.

e-mail:philipp.sterzer@charite.de
In recent years, a number of functional and structural neuroimaging studies have investigated the neural bases of aggressive and violent behaviour in children and adolescents. Most functional neuroimaging studies have persued the hypothesis that pathological aggression is a consequence of deficits in the neural circuits involved in emotion processing. There is converging evidence for abnormal neural responses to emotional stimuli in youths with a propensity towards aggressive behaviour. In addition, recent neuroimaging work has suggested that aggressive behaviour is also associated with abnormalities in neural processes that subserve both the inhibitory control of behaviour and the flexible adaptation of behaviour in accord with reinforcement information. Structural neuroimaging studies in children and adolescents with conduct problems are still scarce, but point to deficits in brain structures in volved in the processing of social information and in the regulation of social and goal-directed behaviour. The indisputable progress that this research field has made in recent years notwithstanding, the overall picture is still rather patchy and there are inconsistencies between studies that await clarification. Despite this, we attempt to provide an integrated view on the neural abnormalities that may contribute to various forms of juvenile aggression and violence, and discuss research strategies that may help to provide a more profound understanding of these important issues in the future.

Keywords: aggression, violence, conduct disorder, fMRI, brain imaging, psychiatry

\section{BACKGROUND}

Despite the undisputable relevance of psychosocial factors and life stressors in understanding aggressive and violent behaviour, the importance of research into the biological basis of pathological aggression has been increasingly acknowledged in recent years (Davidson et al., 2000). Modern brain imaging techniques, such as structural and functional magnetic resonance imaging have been used to investigate the nerual basis of pathological aggression in adults (for review, see McCloskey et al., 2005). While neural abnormalities that can be observed in adults are likely to be the consequence of abnormal behaviour itself and of manifold environmental influences, such as psychosocial factors and substance use, the approach of studying deviant social behaviour early in the course of development maximises the chance of identifying neurobiological mechanisms that are directly related to the its genesis. Moreover, from a clinical perspective, the identification and understanding of the neurobiological mechanisms that give rise to abnormal aggressive behaviour in children will aid the development of specific therapeutic interventions, which are more likely to succeed when applied early in life. Only recently, imaging studies have attempted to characterize neural abnormalities in children and adolescents displaying aggressive or even violent behaviour.

From a psychiatric-diagnostic perspective, abnormal aggressive behaviour in children and adolescents has been subsumed under the diagnoses conduct disorder (CD) and oppositional defiant disorder (ODD) by the the DSM classification system (American Psychiatric Association, 1994). They are characterized by repetitive and chronic aggressive and antisocial behaviour with a variety of implications such as school refusal, social communication problems, and legal involvement. The prevalences of disruptive behaviour disorders are $2 \%$ for CD and 3.2\% for ODD. Boys are more affected than girls (Lahey et al., 1999). There is a high-risk group starting with aggressive behaviour at a particularly young age. This early-onset subtype is characterized by an elevated biological risk and is more likely to take a non-favourable outcome with life-course persistent antisocial behaviour (Moffitt et al., 2008). In contrast, the adolescent-onset subtype has a much better long-term prognosis with relatively unimpaired education, work, health, and family-life outcomes (Moffitt et al., 2008). This is acknowledged to some extent in the DSM-IV by subdividing CD into childhood- and adolescent-onset subtypes (American Psychiatric Association, 1994). While these diagnostic entities may be helpful to guide clinical decision making, it has to be kept in mind that they still represent rather heterogeneous entities and may therefore be of limited use as behavioural phenotypes for the investigation of the neurobiological mechansisms underlying pathological aggression. $\mathrm{CD}$, for example, which is generally considered a more severe disorder than ODD, is not a unitary disorder. First, it is often associated with other co-morbid conditions (Loeber et al., 2000), most notably attention-deficit hyperactivity disorder (ADHD), anxiety disorders, and depression. As CD and ODD are strongly associated with ADHD, a neurobiological overlap between ADHD and disruptive behaviour disorders has been discussed (Banaschewski et al., 2005). Second, CD encompasses a wide range of behavioural abnormalities that may have different neural 
substrates. There is a variety of possible symptoms, ranging from lying and breaking parental rules to more severe offences like rape, assault, mugging, and breaking and entering (American Psychiatric Association, 1994). Moreover, CD can be characterized by different types of aggression, such as impulsive hot-tempered quarrels or premeditated violent acts. A key distinction is between reactive (affective, impulsive or defensive) and instrumental (premeditated and goal-directed) aggression. These two types of aggression have been proposed to differ in their biological basis (Kempes et al., 2005). Reactive aggression is frequent in CD (and antisocial personality disorder in adults) but is also found in other psychiatric disorders, e.g., in bipolar disorder, post-traumatic stress disorder, and very frequently in borderline personality disorder (Blair, 2001, 2003, 2005). Instrumental aggression is more specific for CD but is only observed in a subgroup of CD patients. The propensity for instrumental antisocial behaviour including aggression is characteristic of psychopathic personality, which has been operatinalized as "callous-unemotional traits" in children (Frick et al., 1994). Children with callous-unemotional traits show a specific neurocognitive profile, have more extreme behaviour problems, and a higher genetic risk, suggesting that this subtype of CD is mediated through different psychopathological pathways and different developmental trajectories (Moffitt et al., 2008).

Neuroimaging studies on pathological aggression in children and adolescents have chosen different strategies to define samples of aggressive individuals. While some studies compared groups of individuals fulfilling the diagnostic criteria for CD, others have attempted to disambiguate between "pure" CD and CD with co-morbidities, such as ADHD. Yet another approach that has been used is to focus on subgroups displaying specific subtypes of aggressive behaviour, e.g., boys with callous-unemotional traits. Each of these approaches has its justification and its own merits, but these differences should be kept in mind when interpreting and comparing findings across studies.

\section{EMOTION PROCESSING THEORETICAL BACKGROUND AND BEHAVIOURAL FINDINGS}

It has been proposed that the propensity for aggression and violence is associated with a deficiency in responding to emotional cues in the social environment (Davidson et al., 2000; Herpertz and Sass, 2000; Blair, 2001; Raine, 2002). Several lines of evidence support this notion of a link between emotion processing and aggression. Both children and adults displaying antisocial and violent behaviour show abnormally low autonomic arousal (Blair et al., 1997; Blair, 1999; Raine, 2002; Herpertz et al., 2003, 2005, 2007; Loney et al., 2003). But also the recognition of emotional cues such as sad and fearful facial expressions is impaired in children with antisocial behaviour, especially in those with callous-unemotional traits (Blair et al., 2001; Loney et al., 2003). In addition, emotional shallowness and callousness have been found to be associated with several features of childhood antisocial behaviour (Frick et al., 1994, 2003).

\section{NEURAL ABNORMALITIES IN THE PROCESSING OF AFFECTIVE STIMULI}

Functional MRI (fMRI) studies have examined the neural correlates of emotion processing in aggressive and antisocial adults, especially those with psychopathy (Hare et al., 1991). Functional abnormalities were found in the amygdala, the orbitofrontal cortex (OFC), and the anterior cingulate cortex (ACC) (Kiehl et al., 2001; Veit et al., 2002; Birbaumer et al., 2005). More recently, the neurofunctional correlates of aggressive behaviour were also investigated in children and adolescents (Sterzer et al., 2005; Stadler et al., 2007; Herpertz et al., 2008; Marsh et al., 2008; Jones et al., 2009). The first study on emotion processing in aggressive children and adolescents investigated neural processing of negative affective pictures in a group of boys with CD (Sterzer et al., 2005). This study revealed a negative correlation between aggressive behaviour and responses in the left amygdala to negative affective pictures. The inverse relationship, i.e., a positive correlation was found with symptoms of anxiety and depression, which are often present as co-morbid conditions in CD (Loeber et al., 2000). Consistent with the central role of the amygdala in the processing of stimuli that connote threat, in induced fear, and in generalized negative affect (for review, see Phelps and LeDoux, 2005), this latter finding is in line with the notion of an increased sensitivity to mood-congruent stimuli in negative mood states (Leppanen, 2006). In contrast, the reduced responsiveness of the amygdala in aggressive individuals might reflect deficient emotion processing at the perceptual level as a neural basis for pathological aggression. Viewing of affective pictures was also accompanied by a pronounced deactivation in the ACC in CD patients compared to controls (Sterzer et al., 2005). This abnormal activation was located in the dorsal part of the ACC, which has been implicated in cognitive monitoring and regulation of behaviour rather than emotional processes (Bush et al., 2000). This result may thus indicate an impaired cognitive control of emotional behaviour in patients with $\mathrm{CD}$, in line with the notion of a reciprocal functional relationship between the dorsal and ventralrostral ACC (Drevets and Raichle, 1998; Bush et al., 2000). This interpretation has to remain speculative, as the experimental paradigm used in this study did not explicitly probe cognitive control mechanisms. Interestingly, however, reduced activation in the ACC was associated with the temperament dimension "novelty seeking” (Cloninger, 1987), which comprises high impulsivity and a quick-tempered personality (Stadler et al., 2007). Individuals with high novelty seeking were also characterized by deficient behavioural control strategies and a lower level of socio-moral reasoning (Stadler et al., 2007). The reduced activation in the dorsal ACC of patients with CD might hence be a linking factor between temperament, emotion processing, and behavioural outcome.

A carefully conducted recent study used a similar design to investigate emotion processing in a larger group of boys with CD (Herpertz et al., 2008). These authors found neither a difference in ACC activation nor a correlation between aggressive behaviour and amygdala activation. To the contrary, activation in the left lateral amygdala was found to be even greater in boys with CD compared to healthy conrols. Consistent with the positive correlation of amygdala activation with anxiety and depressive symptoms in the study by Sterzer et al. (2005), this finding could be related to high levels of anxiety and emotional problems in the study by Herpertz et al. (2008), although covariate analyses indicated that amygdala hyperactivation could not be exclusively explained by these symptoms in this latter study. While the severity of anxiety and depressive symptoms were similar in the two studies, levels of aggressive and dissocial behaviour were on average higher in the study by Sterzer et al. (2005), which may have resulted in greater sensitivity for 
neural changes related to these behavioural abnormalities. Another possible explanation for the amygdala hyperactivation in CD found by Herpertz et al. (2008) is that the cluster of increased amygdala activation was located slightly lateral to the cluster where controls showed amygdala activation. The group difference was thus confined to a lateral subregion of the amygdala, and it cannot be ruled out with certainty that greater spatial spread or variability of the amygdala activation in the CD group may have contributed to this finding. Finally, while a similar set of stimuli from the same affective picture data base (Lang et al., 1997) was used in both studies, there were also some differences between the two experimental paradigms: For example, the stimulus set size was considerably smaller and stimuli were presented for shorter durations in the study by Herpertz et al. (2008). This may have hampered the power at the single subject level and hence also overall sensitivity, despite the considerably larger sample size in this study. While these explanations must remain speculative for the moment, the inconsistencies in results clearly call for further studies that should maximise statistical power both by using most efficient stimulation paradigms and, most importantly, by studying larger cohorts.

\section{NEURAL PROCESSING OF DISTRESS CUES}

Two recent studies have addressed the question whether emotion processing is altered in boys with callous-unemotional traits, a subgroup of youths with conduct problems (Marsh et al., 2008; Jones et al., 2009). Both studies investigated the processing of fearful facial expressions using fMRI. They both reported reduced activations in the amygdala of youths with callous-unemotional traits, consistent with the hypothesis that antisocial behaviour in these individuals is related to deficient neural responses to distress cues in others. These reduced amygdala responses could not be accounted for by symptoms of ADHD, which is a frequent co-morbid condition in children with antisocial behaviour, suggesting that the finding is indeed related to antisocial behaviour. Interestingly, functional connectivity between the amygdala and the ventromedial prefrontal cortex is greater in healthy subjects and youths with ADHD relative to those with callous-unemotional traits (Marsh et al., 2008), possibly indicating an inappropriate modulation of instrumental behaviour by stimuli such as others' distress cues.

Deficient responding to distress cues in others suggests an impaired ability to empathize with the feeling of others. A recent fMRI study directly targeted empathy for pain in youths with CD by presenting animated visual stimuli that showed other people experiencing pain (Decety et al., 2009). Surprisingly, this preliminary report of a small group of subjects ( $n=8$ per group) showed greater activations in the amygdala and other temporal and prefrontal regions of youths with CD compared to healthy control participants when they viewed others in pain, compared to no pain. Amygdala responses in CD youths correlated with their parents' ratings of daring behaviour and sadism, which may indicate that increased amygdala responses could reflect a positive affective response (excitement or enjoyment) to seeing others in pain. In line with the above mentioned study by Marsh et al. (2008), analyses of functional connectivity revealed that adolescents with CD exhibited less coupling between the amygdala and prefrontal cortical regions, suggesting impaired regulatory interactions between prefrontal cortex and the amygdala.

\section{BEHAVIOUR REGULATION MOTIVATIONAL REGULATION OF BEHAVIOUR}

In addition to abnormal emotion processing and related deficits in empathy, repetitive antisocial behaviour despite the prospect or even the direct experience of punishment or other negative consequences may be related to motivational deficits (Blair, 2004). Indeed, children with disruptive behaviour problems show abnormalities in motivational regulation of behaviour (Matthys et al., 2004). Moreover, both children and adults with psychopathic traits are impaired in reversal learning tasks (Budhani and Blair, 2005; Budhani et al., 2006), which index the ability to flexibly adjust to changing reinforcement contigencies. In healthy individuals, the ventromedial prefrontal cortex has been implicated in reversal learning (Cools et al., 2002; O'Doherty et al., 2003). A recent study used fMRI to compare the neural basis of reversal learning in children with callous-unemotional traits with ADHD patients and healthy controls (Finger et al., 2008). Children with psychopathic traits showed increased responses in ventromedial prefrontal cortex during punished reversal errors compared to both ADHD children and healthy controls. This abnormal activation in ventromedial prefrontal cortex suggests that children with psychopathic traits may not appropriately process the violation of reinforcement expectations. Ventromedial prefrontal cortex is known to play a role in the computation of reward expectations (Elliott and Deakin, 2005).Violation of these expectations can lead to frustration, which has long been linked to reactive aggression (Berkowitz, 1993). As ventromedial prefrontal cortex is involved in the computations of expectation violation that are necessary to induce frustration, the increased activity in this region during reversal errors may reflect a signal involved in triggering reactive aggression under conditions where an expected reward has not been achieved. Exaggerated ventromedial prefrontal responses to expectation violations may thus form the neural basis for an increased risk for frustration that gives in turn rise to an increased risk for aggressive behaviour (Blair, 2009).

A different aspect of reward processing was investigated by a recent fMRI study that sought to dissociate dysfunctions in attention and reward mechanims in boys with ADHD and CD, respectively (Rubia et al., 2009b). These authors used a sustainedattention paradigm, the continuous performance task, where participants had to respond to targets that were either associated with a monetary reward or not. Boys with "pure" CD (i.e., no comorbid ADHD) showed reduced activation during rewarded trials in lateral OFC, compared to boys with pure ADHD (no co-morbid CD) and healthy controls. This finding was interpreted as reflecting hyporesponsiveness to reward cues. However, how such hyporesponsiveness relates to aggressive or antisocial behaviour is currently unclear. The lateral subregion of OFC has been implicated in the evaluation of punishment (versus reward) values of stimuli and is thought to thus provide a signal that can lead to a change in current behaviour (Kringelbach and Rolls, 2004). Of course, the link between dysfunction in a brain region involved in punishment processing and aggressive or antisocial behaviour has some intuitive plausibility. However, a deeper understanding of this relationship will require further investigation with experiments that specifically address the question how orbitofrontal function relates to the processing of punishment in antisocial individuals. 
Taken together the two recent studies by Finger et al. (2008) and Rubia et al. (2009b) provide first evidence for deficits in prefrontal brain regions crucially involved in motivational control of behaviour, possibly indicating impairments in reinforcementguided adjustment of behaviour. These functional neural deficits may be related to structural abnormalities in prefrontal cortex (Huebner et al., 2008; De Brito et al., 2009) that are discussed in more detail below.

\section{ATTENTION AND INHIBITORY CONTROL}

Aggressive and antisocial behaviour, especially reactive aggression, has been linked to impulsivity, that is, to deficits in restraining immediate behavioural responses in accord with anticipated longterm consequences (Berkowitz, 2008). Clinically, this notion is supported by a large overlap between CD and ADHD (Loeber et al., 2000). Also in ADHD impulsivity is a core feature, in addition to inattention and hyperactivity (American Psychiatric Association, 1994). Two recent studies have asked whether CD and ADHD share common neural deficits in the inhibitory control of behaviour and whether there are neural abnormalities reflecting a deficit in inhibitory control that may be specific to CD rather than ADHD (Rubia et al., 2008, 2009a). Rubia et al. (2008) measured the neural correlates of inhibition and stopping failure during a stop task in a group of boys with pure CD compared to boys with pure ADHD and a group of healthy controls using fMRI. They found that the neural abnormalities related to successful response inhibition involved mainly dorsolateral prefrontal cortex, both in CD and ADHD. In contrast, activations in response to inhibition failures were reduced in bilateral temporoparietal cortex in boys with CD but not in ADHD. Of note, the participants received implicit feedback during inhibition failures, such that fMRI activations during inhibition failures most likely reflect error detection and subsequently enhanced functions of arousal and attention allocation to the committed error. Reduced responses in temporoparietal cortex, which has been implicated in the detection of behaviourally relevant events (Corbetta and Shulman, 2002), may therefore reflect deficient performance monitoring in youths with CD. In other words, individuals with $\mathrm{CD}$ may be less alerted by their mistakes and be therefore less capable of adjusting their behaviour to negative feedback, as has also been shown behaviourally (Matthys et al., 2004; van Goozen et al., 2004).

In a second study from the same laboratory (Rubia et al., 2009a), fMRI was used to study the neural substrates of inference inhibition, again in boys with either CD or ADHD compared to healthy controls. They used a classical Simon task (Simon, 1969) where spatial information interferes with iconic information of the target stimuli, combined with an oddball task (involving the occasional presentation of deviant stimuli), to allow for the dissociation of neural signals related to inference inhibition from those related to selective attention. This study showed shared deficits in right superior temporal cortex during inference inhibition and in the precuneus and right medial prefrontal cortex during the oddball condition, in both CD and ADHD. While there was a specific deficit associated with oddballs in left ventrolateral prefrontal cortex in the ADHD group, no disorder-specific deficits were found in the $\mathrm{CD}$ group using this paradigm.
Together, recent work investigating the neural substrates of attention and inhibitory control shows a large overlap in functional brain abnormalities in ADHD an CD. However, there are also deficits that appear to be specific to CD. These deficits are related to inhibition failures and may reflect a specific impairment in response monitoring and learning from negative feedback in youths with CD.

\section{STRUCTURAL ABNORMALITIES}

Recent structural MRI studies that used voxel-based morphometry (VBM), an automated voxel-wise method for the detection of regional differences in grey or white matter (Ashburner and Friston, 2000), have provided confirmatory evidence for neural deficits in brain structures that showed functional abnormalities in $\mathrm{fMRI}$ studies. Two VBM investigations in youth with CD showed decreased grey-matter volume in mesial temporal lobe structures, including the amygdala, and a significant association of these greymatter deficits with the severity of conduct problems (Sterzer et al., 2007; Huebner et al., 2008). Orbitofrontal grey-matter volume was also found to be reduced in youth with CD (Huebner et al., 2008), in line with previous reports of conduct problems in individuals with early acquired prefrontal lobe damage (Anderson et al., 1999) and thus supporting the notion that OFC plays an important role in regulating aggressive behaviour. Sterzer et al. (2007) additionally found a grey-matter volume reduction in bilateral anterior insular cortex. Interstingly, anterior insular grey-matter volume in CD patients was positively correlated with empathy levels as assessed behaviourally, in line with recent evidence from fMRI suggesting a crucial involvement of the anterior insula in the experience of empathy (de Vignemont and Singer, 2006). Somewhat surprisingly, a VBM study in a non-clinical sample of boys with callous-unemotional traits reported increased instead of decreased grey-matter in orbitofrontal and anterior cingulate cortices (De Brito et al., 2009). This finding could be explained by a delay in cortical maturation, as the physiological prefrontal age-related grey-matter decrease found in normally developing children was not observed in the group of callous-unemotional boys. The inconsistencies in findings are most likely related to differences in sample characteristics between the study by De Brito et al. (2009) and the other two VBM studies by Sterzer et al. (2007) and Huebner et al. (2008). While De Brito et al. (2009) studied a non-clinical sample with callous-unemotional traits, the other two studies investigated clinical samples with CD, but did not differentiate whether their children with conduct problems had callous-unemotional traits or not. Moreover, compared to the other two studies, the participants were on average younger in the study by De Brito et al. (2009); and the age range in this study was narrower (10-13 years) and spanned the most critical period for prefrontal development, which could have increased sensitivity for differences related to developmental delay. Clearly, there is a need for further research to explain the seemingly opposing results of these studies.

\section{GENERAL DISCUSSION AND OUTLOOK}

In recent years, there has been a surge in brain imaging studies that attempted to elucidate the neural basis of aggressive and antisocial behaviour as early as in childhood and adolescence. Under the hypothesis that these behavioural abnormalities may result from 
deficits in recognizing emotional expressions, especially distress cues, in others and from a dysregulation of emotional behaviour, a number of brain imaging studies have focused on deficits in emotion processing. Other studies have highlighted abnormalities in motivational processes and in inhibitory control of behaviour, thereby extending our knowledge about possible neural mechanisms that may underlie aggression and violence early in life. It should be kept in mind that this research area is still in its infancy and that more research is needed in order to develop a comprehensive model of the neural mechanisms underlying juvenile antisocial behaviour (see below). Nevertheless, it seems justified to summarize the findings from neuroimaging studies so far in a tentative and - at this stage - descriptive way (Figure 1).

There seems to be converging evidence for a dysfunction of limbic structures, first and foremost the amygdala (Sterzer et al., 2005, 2007; Herpertz et al., 2008; Huebner et al., 2008; Marsh et al., 2008; Decety et al., 2009; Jones et al., 2009). Reduced responsiveness of the amygdala to fearful faces (Marsh et al., 2008; Jones et al., 2009) may reflect an impairment in recognizing distress cues in others. According to the violence-inhibition mechanism model (Blair, 2001), this could in turn lead to a lack of empathy and deficient control of aggressive behaviour. Alternatively, reduced amygdala responsiveness could also be interpreted as reflecting a reduced sensitivity to stimuli connotating threat, in line with the notion that preferential amygdala responses to fearful faces subserve the modulation of vigilance in threatening situations rather than the recognition of social cues (Davis and Whalen, 2001). It should be noted that some studies reported hyperresponsiveness of the amygdala (Herpertz et al., 2008; Decety et al., 2009), depending on the task used and the behavioural characteristics of the sample studied. It cannot therefore be concluded that aggressive and antisocial behaviour is related to a general reduction in amygdala responsiveness. Conceivably, in the same group of antisocial indivuals, the amygdala may be hyporesponsive when it comes to emotion recognition or threat detection, but hyperresponsive in relation to excitement, e.g., about the brutality of a situation (Decety et al., 2009). Moreover, the amygdala may be differentially involved in aggressive behaviour resulting from anxiety (Sterzer et al., 2005; Herpertz et al., 2008) as opposed to aggression due to emotional callousness (Marsh et al., 2008; Jones et al., 2009). It is an intriguing challenge for future research to provide a more sophisticated picture of how dysregulation of amygdala function contributes to aggressive behaviour.

In addition to the amygdala, there is also evidence for dysfunction in prefrontal brain regions, most importantly orbitofrontal and ventromedial prefrontal cortices (Finger et al., 2008; Huebner et al., 2008; Marsh et al., 2008; Rubia et al., 2008; De Brito et al., 2009; Decety et al., 2009), but also the dorsal ACC (Sterzer et al., 2005; De Brito et al., 2009). A possible interpretation of these findings is that dysfunction of prefrontal circuits may underlie an impaired regulation of affective responses, resulting in a reduced ability to restrain aggressive responses (Davidson et al., 2000; Blair, 2001). Moreover abnormal function in orbitofrontal and ventromedial prefrontal cortices was also observed during reinforcement processing (Finger et al., 2008; Rubia et al., 2008), which may indicate deficient learning mechanisms with regard to social rules (Adolphs, 2003; Blair, 2004). Finally, the considerable clinical overlap between CD and ADHD is reflected by a large overlap in functional neural deficits during attention allocation and inhibitory control of behaviour (Rubia et al., 2008, 2009a,b), but there are specific functional abnormalities in temporoparietal cortex of youths with CD that may be related to impaired learning from negative feedback (Rubia et al., 2008).

Despite the considerable progress in the understanding of juvenile pathological aggression and violence, it should be noted that evidence from neuroimaging is still relatively scarce. There are

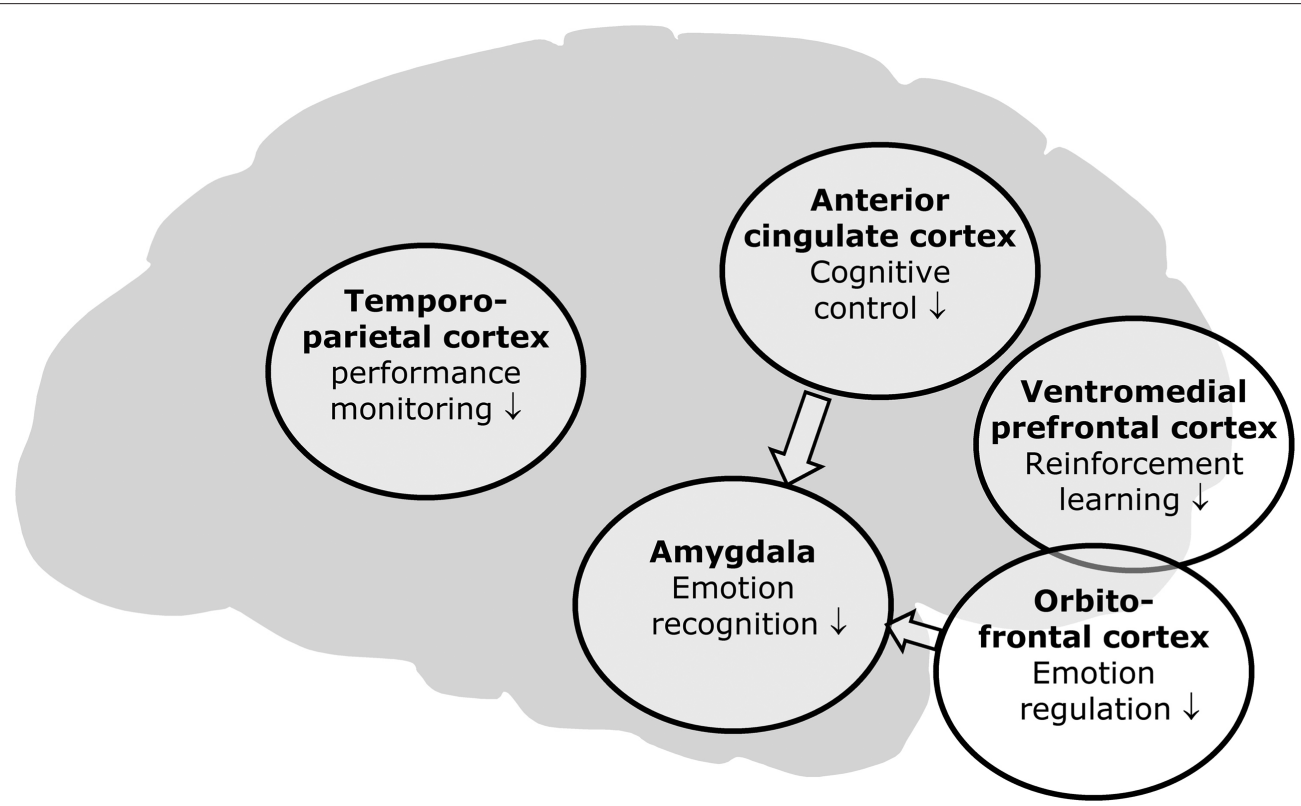

FIGURE 1 | Summary of neuroimaging findings related to aggressive and violent behaviour in children and adolescents. 
inconsistencies between studies that cannot be readily explained at the moment, and some of the findings await replication before strong conclusions should be made. One obvious problem is that the sample sizes in most studies are relatively small $(n=8-24)$. Most studies reviewed here applied state-of-the-art statistical methods and it is therefore reasonable to assume that their results are in principle valid. However, the known heterogeneity of aggressive behaviours is likely to give rise to qualitatively different results across studies, which makes it difficult to draw strong general conclusions. As mentioned above, the heterogeneity is reflected by the fact that juvenile aggressive and antisocial behaviour, which is usually diagnosed as CD, is often associated with other co-morbid conditions (Loeber et al., 2000), most notably ADHD and mood disorders. This alone implies that aggressive behaviour, even though phenotypically similar, may be based on different pathomechanisms. For example, bursts of reactive aggression may be merely due to a deficit in impulse control, but could also be based on anxiety or negative mood; or undamped reactive aggression could result from callous-unemotional traits, just because there is no insight into the necessity to comply with social rules and to control one's aggressive impulses accordingly. As this example shows, it seems essential to carefully characterize the study populations with respect to both the phenotypical expression of behaviour (e.g., reactive vs. instrumental aggression) and accompanying conditions, such as psychiatric co-morbitidities. In addition, considering early temperamental risk factors might also be helpful in identifying subgroups of patients with distinct neurobiological profiles. For example, children with callous-unemotional traits show a specific neural profile suggestive of amygdala hypofunction, as manifested by insensitivity to fearful facial expressions (Marsh et al., 2008; Jones et al., 2009). This profile seems to differ from that of other children with $\mathrm{CD}$, in whom even increased amygdala responses to affective information have been observed (Herpertz et al., 2008; Decety et al., 2009). Finally, neuroimaging of juvenile aggression and violence will undoubtedly also benefit from the incorporation of genetic factors that may predispose towards pathological

\section{REFERENCES}

Adolphs, R. (2003). Cognitive neuroscience of human social behaviour. Nat. Rev. Neurosci. 4, 165-178.

American Psychiatric Association (1994). Diagnostic and Statistical Manual of Mental Disorders, DSM-IV, 4th Edn. Washington, DC,American Psychiatric Association.

Anderson, S. W., Bechara, A., Damasio, H., Tranel, D., and Damasio, A. R. (1999). Impairment of social and moral behaviour related to early damage in human prefrontal cortex. Nat. Neurosci. 2, 1032-1037.

Ashburner, J., and Friston, K. J. (2000). Voxel-based morphometry-the methods. Neuroimage 11, 805-821.

Banaschewski, T., Hollis, C., Oosterlaan, J., Roeyers, H., Rubia, K., Willcutt, E., and Taylor, E. (2005). Towards an understanding of unique and shared 27, 135-145. aggression. This approach offers the opportunity to elucidate how genetic and environmental factors interact to give rise to aberrant social behaviours (Viding and Jones, 2008).

It is a great challenge for the promising field of neuroimaging research into the neurobiology of aggression and violence to come to grips with the diversity of phenotypic expressions of this behaviour on the one hand, and the multitude of potential pathogenetic factors on the other hand. One possible strategy is to try and isolate subgroups with distinct phenotypical features, especially in cases of strong and concise a priori hypotheses concerning the mechanism underlying a specific type of behaviour. The inherent problem of this approach could be that behavioural abnormalities usually do not come in clear-cut, pure categories, and that the artificial separation in such categories may hamper the interpretation and "real-life" application of the resulting findings. Another possible strategy is to aim at large study cohorts that may comprise various subtypes of aggressive behaviour and also allow for genotypic variability. In that case, the problem of heterogeneity must be dealt with at the stage of data analysis using dimensional or factorial approaches. Finally, longitudinal studies in large community-based samples or in clinical populations will be indispensable for a more complete pathophysiological understanding of the development, timecorse and prognosis of aggression and violence during childhood and adolescence. Neuroimaging may provide useful prognostic information or surrogate markers for treatment monitoring, for example based on the use of novel pattern-based approaches for the analysis of neuroimaging data (Haynes and Rees, 2006). The choice of research strategy of course depends on the precise scientific question asked and the hypothesis posed, but we believe that the strategies proposed here have the potential to further our understanding of juvenile pathological aggression and violence in the future.

\section{ACKNOWLEDGMENTS}

Philipp Sterzer is supported by Deutsche Forschungsgemeinschaft (Emmy-Noether Program, STE-1430/2-1).
J. Neurol. Neurosurg. Psychiatry 71, 727-731.

pathways in the psychopathophysiology of ADHD. Dev. Sci. 8, 132-140.

Berkowitz, L. (1993). Aggression: Its Causes, Consequences, and Control. Philadelphia, Temple University Press.

Berkowitz, L. (2008). On the consideration of automatic as well as controlled psychological processes in aggression. Aggress. Behav. 34, 117-129.

Birbaumer, N., Veit, R., Lotze, M., Erb, M., Hermann, C., Grodd, W., and Flor, H. (2005). Deficient fear conditioning in psychopathy: a functional magnetic resonance imaging study. Arch. Gen. Psychiatry 62, 799-805.

Blair, R. J. (1999). Responsiveness to distress cues in the child with psychopathic tendencies. Pers. Individ. Dif.

Blair, R. J. (2001). Neurocognitive models of aggression, the antisocial personality disorders, and psychopathy.
Blair, R. J. (2003). Neurobiological basis of psychopathy. Br. J. Psychiatry 182, 5-7.

Blair, R. J. (2004). The roles of orbital frontal cortex in the modulation of antisocial behavior. Brain Cogn. 55 198-208.

Blair, R. J. (2005). Responding to the emotions of others: Dissociating forms of empathy through the study of typical and psychiatric populations. Conscious Cogn. 14, 698-718.

Blair, R. J. (2009). Psychopathy, frustration, and reactive aggression: the role of ventromedial prefrontal cortex. Br. J. Psychol. [Epub ahead of print].

Blair, R. J., Colledge, E., Murray, L., and Mitchell, D. G. (2001). A selective impairment in the processing of sad and fearful expressions in children with psychopathic tendencies.
J. Abnorm. Child Psychol. 29 491-498.

Blair, R. J., Jones, L., Clark, F., and Smith, M. (1997). The psychopathic individual: a lack of responsiveness to distress cues? Psychophysiology 34, 192-198.

Budhani, S., and Blair, R. J. (2005). Response reversal and children with psychopathic tendencies: success is a function of salience of contingency change. J. Child Psychol. Psychiatry 46, 972-981.

Budhani, S., Richell, R. A., and Blair, R. J. (2006). Impaired reversal but intact acquisition: probabilistic response reversal deficits in adult individuals with psychopathy. J. Abnorm. Psychol. 115, 552-558.

Bush, G., Luu, P., and Posner, M. I. (2000). Cognitive and emotional influences in anterior cingulate cortex. Trends Cogn. Sci. 4, 215-222. 
Cloninger, C. R. (1987). A systematic method for clinical description and classification of personality variants. A proposal. Arch. Gen. Psychiatry 44, 573-588.

Cools, R., Clark, L., Owen, A. M., and Robbins, T. W. (2002). Defining the neural mechanisms of probabilistic reversal learning using event-related functional magnetic resonance imaging. J. Neurosci. 22, 4563-4567.

Corbetta, M., and Shulman, G. L. (2002). Control of goal-directed and stimulusdriven attention in the brain. Nat. Rev. Neurosci. 3, 201-215.

Davidson, R. J., Putnam, K. M., and Larson, C. L. (2000). Dysfunction in the neural circuitry of emotion regulation-a possible prelude to violence. Science 289, 591-594.

Davis, M., and Whalen, P. J. (2001). The amygdala: vigilance and emotion. $\mathrm{Mol}$. Psychiatry 6, 13-34.

De Brito, S. A., Mechelli, A., Wilke, M., Laurens, K. R., Jones, A. P., Barker, G. J., Hodgins, S., and Viding, E. (2009). Size matters: increased grey matter in boys with conduct problems and callous-unemotional traits. Brain 132, 843-852.

de Vignemont, F., and Singer, T. (2006). The empathic brain: how, when and why? Trends Cogn. Sci. 10, 435-441.

Decety, J., Michalska, K. J., Akitsuki, Y., and Lahey, B. B. (2009). Atypical empathic responses in adolescents with aggressive conduct disorder: a functional MRI investigation. Biol. Psychol. 80, 203-211.

Drevets, W. C., and Raichle, M. E. (1998). Reciprocal suppression of regional cerebral blood flow during emotional versus higher cognitive processes: implications for interactions between emotion and cognition. Cogn. Emot. 12, 353-385.

Elliott, R., and Deakin, B. (2005). Role of the orbitofrontal cortex in reinforcement processing and inhibitory control: evidence from functional magnetic resonance imaging studies in healthy human subjects. Int. Rev. Neurobiol. 65, 89-116.

Finger, E. C., Marsh, A.A., Mitchell, D. G., Reid, M. E., Sims, C., Budhani, S., Kosson, D. S., Chen, G., Towbin, K. E., Leibenluft, E., Pine, D. S., and Blair, J. R. (2008). Abnormal ventromedial prefrontal cortex function in children with psychopathic traits during reversal learning. Arch. Gen. Psychiatry 65, 586-594.

Frick, P. J., Cornell, A. H., Bodin, S. D., Dane, H. E., Barry, C. T., and Loney, B. R. (2003). Callousunemotional traits and developmental pathways to severe conduct problems. Dev. Psychol. 39, 246-260.
Frick, P. J., O’Brien, B. S., Wootton, J. M., and McBurnett, K. (1994). Psychopathy and conduct problems in children. J. Abnorm. Psychol. 103, 700-707.

Hare, R. D., Hart, S. D., and Harpur, T. J. (1991). Psychopathy and the DSM-IV criteria for antisocial personality disorder. J. Abnorm. Psychol. 100, 391-398.

Haynes, J. D., and Rees, G. (2006). Decoding mental states from brain activity in humans. Nat. Rev. Neurosci. 7, 523-534.

Herpertz, S. C., Huebner, T., Marx, I., Vloet, T. D., Fink, G. R., Stoecker, T., Shah, N. J., Konrad, K., and HerpertzDahlmann, B. (2008). Emotional processing in male adolescents with childhood-onset conduct disorder. J. Child Psychol. Psychiatry 49, 781-791.

Herpertz, S. C., Mueller, B., Qunaibi, M., Lichterfeld, C., Konrad, K., and Herpertz-Dahlmann, B. (2005). Response to emotional stimuli in boys with conduct disorder. Am. J. Psychiatry 162, 1100-1107.

Herpertz, S. C., Mueller, B., Wenning, B., Qunaibi, M., Lichterfeld, C., and Herpertz-Dahlmann, B. (2003). Autonomic responses in boys with externalizing disorders. J. Neural Transm. 110, 1181-1195.

Herpertz, S. C., and Sass, H. (2000). Emotional deficiency and psychopathy. Behav. Sci. Law 18, 567-580.

Herpertz, S. C., Vloet, T., Mueller, B., Domes, G., Willmes, K., and HerpertzDahlmann, B. (2007). Similar autonomic responsivity in boys with conduct disorder and their fathers. J. Am. Acad. Child Adolesc. Psychiatry 46, 535-544.

Huebner, T., Vloet, T. D., Marx, I., Konrad, K., Fink, G. R., Herpertz, S. C. and Herpertz-Dahlmann, B. (2008). Morphometric brain abnormalities in boys with conduct disorder. J. Am. Acad. Child Adolesc. Psychiatry 47, 540-547.

Jones, A. P., Laurens, K. R., Herba, C. M. Barker, G. J., and Viding, E. (2009). Amygdala hypoactivity to fearful faces in boys with conduct problems and callous-unemotional traits. Am. J. Psychiatry 166, 95-102.

Kempes, M., Matthys, W., de Vries, H., and van Engeland, H. (2005). Reactive and proactive aggression in children-a review of theory, findings and the relevance for child and adolescent psychiatry. Eur. Child Adolesc. Psychiatry 14, 11-19.

Kiehl, K. A., Smith, A. M., Hare, R. D., Mendrek, A., Forster, B. B., Brink, J., and Liddle, P. F. (2001). Limbic abnormalities in affective processing by criminal psychopaths as revealed by functional magnetic resonance imaging. Biol. Psychiatry 50, 677-684.
Kringelbach, M.L., and Rolls, E. T. (2004). The functional neuroanatomy of the human orbitofrontal cortex: evidence from neuroimaging and neuropsychology. Prog. Neurobiol. 72, 341-372.

Lahey, B. B., Goodman, S. H., Waldman, I. D., Bird, H., Canino, G. Jensen, P., Regier, D., Leaf, P. J., Gordon, R., and Applegate, B. (1999). Relation of age of onset to the type and severity of child and adolescent conduct problems. J. Abnorm. Child Psychol. 27, 247-260.

Lang, P. J., Bradley, M. M., and Cuthbert, B. N. (1997). International Affective Picture System (IAPS): Technical Manual and Affective Ratings. Gainsville, FL, NIMH Center for the Study of Emotion and Attention, University of Florida.

Leppanen, J. M. (2006). Emotional information processing in mood disorders: a review of behavioral and neuroimaging findings. Curr. Opin. Psychiatry 19, 34-39.

Loeber, R., Burke, J. D., Lahey, B. B., Winters, A., and Zera, M. (2000). Oppositional defiant and conduct disorder: a review of the past 10 years, part I. J. Am. Acad. Child Adolesc. Psychiatry 39, 1468-1484.

Loney, B. R., Frick, P. J., Clements, C. B. Ellis, M. L., and Kerlin, K. (2003). Callous-unemotional traits, impulsivity, and emotional processing in adolescents with antisocial behaviour problems. J. Clin. Child Adolesc. Psychol. 32, 66-80.

Marsh, A. A., Finger, E. C., Mitchell, D. G. Reid, M. E., Sims, C., Kosson, D. S., Towbin, K. E., Leibenluft, E., Pine, D. S. and Blair, R. J. (2008). Reduced amygdala response to fearful expressions in children and adolescents with callousunemotional traits and disruptive behavior disorders. Am. J. Psychiatry 165, 712-720.

Matthys, W., van Goozen, S. H., Snoek, H., and van Engeland, H.(2004). Response perseveration and sensitivity to reward and punishment in boys with oppositional defiant disorder. Eur. Child Adolesc. Psychiatry 13, 362-364.

McCloskey, M. S., Phan, K. L., and Coccaro, E. F. (2005). Neuroimaging and personality disorders. Curr. Psychiatry Rep. 7, 65-72.

Moffitt, T. E., Arseneault, L., Jaffee, S. R., Kim-Cohen, J., Koenen, K. C., Odgers, C. L., Slutske, W. S., and Viding, E. (2008). Research review: DSM-V conduct disorder: research needs for an evidence base. J. Child Psychol. Psychiatry 49, 3-33.

O’Doherty,J.,Critchley,H.,Deichmann, R. and Dolan, R. J. (2003). Dissociating valence of outcome from behavioural control in human orbital and ventral prefrontal cortices. J. Neurosci. 23, 7931-7939.

Phelps, E. A., and LeDoux, J. E. (2005). Contributions of the amygdala to emotion processing: from animal models to human behaviour. Neuron 48, 175-187.

Raine, A. (2002). Annotation: the role of prefrontal deficits, low autonomic arousal, and early health factors in the development of antisocial and aggressive behaviour in children. J. Child Psychol. Psychiatry 43, 417-434.

Rubia, K., Halari, R., Smith, A. B., Mohammad, M., Scott, S., and Brammer, M. J. (2009a). Shared and disorder-specific prefrontal abnormalities in boys with pure attention-deficit/hyperactivity disorder compared to boys with pure CD during interference inhibition and attention allocation. J. Child Psychol. Psychiatry 50, 669-678.

Rubia, K., Smith, A. B., Halari, R., Matsukura, F., Mohammad, M., Taylor, E., and Brammer, M. J.(2009b). Disorder-specific dissociation of orbitofrontal dysfunction in boys with pure conduct disorder during reward and ventrolateral prefrontal dysfunction in boys with pure ADHD during sustained attention. Am. J. Psychiatry 166, 83-94.

Rubia, K., Halari, R., Smith, A. B., Mohammed, M., Scott, S. Giampietro, V., Taylor, E., and Brammer, M. J. (2008). Dissociated functional brain abnormalities of inhibition in boys with pure conduct disorder and in boys with pure attention deficit hyperactivity disorder. Am. J. Psychiatry 165, 889-897.

Simon, J. R. (1969). Reactions toward the source of stimulation. J. Exp. Psychol. 81, 174-176.

Stadler, C., Sterzer, P., Schmeck, K., Krebs, A., Kleinschmidt, A., and Poustka, F. (2007). Reduced anterior cingulate activation in aggressive children and adolescents during affective stimulation: association with temperament traits. J. Psychiatr. Res. 41, 410-417.

Sterzer, P., Stadler, C., Krebs, A., Kleinschmidt, A., and Poustka, F. (2005).Abnormal neural responses to emotional visual stimuli in adolescents with conduct disorder. Biol. Psychiatry 57, 7-15.

Sterzer, P., Stadler, C., Poustka, F., and Kleinschmidt, A. (2007). A structural neural deficit in adolescents with conduct disorder and its association with lack of empathy. Neuroimage 37, 335-342.

van Goozen, S. H., Cohen-Kettenis, P. T., Snoek, H., Matthys, W., SwaabBarneveld, H., and van Engeland, H. 
(2004). Executive functioning in children: a comparison of hospitalised ODD and ODD/ADHD children and normal controls. J. Child Psychol. Psychiatry 45, 284-292.

Veit, R., Flor, H., Erb, M., Hermann, C., Lotze, M., Grodd, W., and Birbaumer, N. (2002). Brain circuits involved in emotional learning in antisocial behavior and social phobia in humans. Neurosci. Lett. 328, 233-236.

Viding, E., and Jones, A. P. (2008). Cognition to genes via the brain in the study of conduct disorder. Q. J. Exp. Psychol. (Colchester) 61, 171-181.

Conflict of Interest Statement: The authors declare that this article was written in the absence of any commercial or financial relationships that could be construed as a potential conflict of interest.

Received: 30 July 2009; paper pending published: 13 August 2009; accepted: 25 September 2009; published online: 12 October 2009.

Citation: Sterzer P and Stadler C (2009) Neuroimaging of aggressive and violent behaviour in children and adolescents.
Front. Behav. Neurosci. 3:35. doi: 10.3389/neuro.08.035.2009

Copyright (C) 2009 Sterzer and Stadler. This is an open-access article subject to an exclusive license agreement between the authors and the Frontiers Research Foundation, which permits unrestricted use, distribution, and reproduction in any medium, provided the original authors and source are credited. 